\title{
PRESENCIA Y TRATAMIENTO DE LA CULTURA: LA COMPETENCIA INTERCULTURAL Y EL HUMOR EN LOS MANUALES DE ELE UTILIZADOS EN JAPÓN
}

\author{
Carlos Manadé-Rodríguez y Alicia Rodríguez-Álvarez \\ Universidad de Las Palmas de Gran Canaria \\ Instituto Universitario de Análisis y Aplicaciones Textuales (IATEXT)
}

\section{Resumen}

El trabajo propuesto analiza cinco manuales de ELE empleados en Japón con el fin de valorar (a) la forma de integrar los contenidos culturales en estos volúmenes, (b) el tipo de contenidos culturales que reciben más atención (siguiendo la clasificación de Miquel y Sans, 1992), (c) el desarrollo de la competencia intercultural y, finalmente, (d) la presencia de un elemento sociocultural que se manifiesta de muy distintas formas en las culturas observadas: el humor. Los resultados revelarán, por un lado, algunos patrones comunes en cuanto al modo de integrar estos contenidos en los manuales, y, por otro, diferencias en la tipología de los contenidos tratados y la promoción del espíritu crítico asociado a la competencia intercultural. Por último, resulta muy llamativo el nulo interés que se presta a las manifestaciones verbales del humor, un elemento muy presente en el español coloquial y causante de muchos malentendidos en las interacciones sociales.

Palabras clave: ELE, manuales, contenidos culturales, competencia intercultural, humor.

\section{THE PRESENCE OF AND APPROACH TO CULTURE: INTERCULTURAL COMPETENCE AND HUMOUR IN SFL HANDBOOKS IN JAPAN}

\begin{abstract}
This work analyses five SFL handbooks used in Japan in order to assess (a) how cultural contents are included in these handbooks, (b) the type of cultural content that receives more attention (following the classification of Miquel and Sans, 1992), (c) the development of intercultural competence, and, finally, (d) the presence of a sociocultural element that varies greatly across cultures: humour. The results will identify some common patterns as for the way these handbooks include cultural contents, but they will also reveal differences in the typology of the contents handled and the promotion
\end{abstract}

Fecha de recepción: 19 de marzo de 2018

Fecha de aceptación: 28 de junio de 2018 
of the critical spirit associated with intercultural competence. Finally, considering the extensive presence of humour in colloquial Spanish and its role as the cause of many misunderstandings in social interactions, it is noteworthy that no attention has been paid to this element so far.

Keywords: SFL, handbooks, cultural contents, intercultural competence, humour.

\section{IntroducGión}

La influencia de los manuales en la praxis docente permite deducir que, a partir del análisis del contenido que incluyen y las destrezas que desarrollan, se puede llegar a anticipar el tipo de enseñanza que llevará a cabo el profesor que los utilice (Venezky, 1992: 436; Paricio Tato, 2005: 138). En el presente estudio nos proponemos analizar cinco manuales de español como lengua extranjera (ELE) empleados en Japón para determinar la presencia y el tratamiento de la cultura, la competencia intercultural y, como expresión particular de estas, el humor verbal en la enseñanza de ELE en ese país. La elección del humor como elemento sociocultural de especial relevancia responde a su extendida presencia en el español coloquial (Beinhauer, 1973: 12).

Para que el alumnado japonés de ELE sepa desenvolverse en contextos socioculturales que le son extraños, es crucial que, junto al conocimiento lingüístico, aprenda los comportamientos, las costumbres, las actitudes y otras características propias de la cultura española y latinoamericana. Sin embargo, no basta con esto, pues la adquisición de este nuevo conocimiento debe ir acompañada del desarrollo de una actitud crítica y reflexiva, es decir, de la competencia intercultural. Dentro del saber cultural general, el humor juega un papel fundamental, pues es un mecanismo de gran aplicación social y con múltiples funciones en la interacción. Sin embargo, aunque la utilidad del humor es universal, sus manifestaciones lingüísticas varían. Ciertamente, como señala González Verdejo (2003: 347), «el humor se nos presenta como reflejo de las particularidades de cada pueblo y cultura. Es precisamente este último aspecto el que lo convierte en una herramienta didáctica de gran valía para desarrollar la competencia intercultural de nuestros alumnos»; por eso, Bell y Pomerantz (2016: 169) defienden que el humor debería enseñarse como una dimensión pragmática más de las lenguas extranjeras.

Así pues, teniendo en cuenta la importancia de estos tres elementos en el acto comunicativo, en el estudio de los manuales propuesto nos hemos marcado los siguientes objetivos:

- Describir cómo incorporan el componente cultural.

- Señalar qué tipo de contenido cultural integran. 
- Analizar el grado de desarrollo de la competencia intercultural.

- Examinar el espacio dedicado al humor y a las estrategias para verbalizarlo.

Además, este análisis nos permitirá identificar diferencias en el planteamiento y contenidos de aquellos materiales elaborados para estudiantes de ELE en general y aquellos específicos para estudiantes japoneses; pero, antes de abordar estas cuestiones, es necesario precisar qué entendemos en este trabajo por 'cultura' y por 'competencia intercultural' y cuál es el registro humorístico que se analiza en estos manuales.

\subsection{Los contenidos (inter)culturales en la enseñanza de ELE}

El desarrollo de la competencia comunicativa intercultural propuesta por Byram (1997) supuso una evolución de la competencia cultural o sociocultural de Van Ek (1986). Esta nueva competencia permite al aprendiente desempeñar la función de mediador entre las culturas en contacto, incluyendo la suya propia, siendo consciente de que todo «encuentro intercultural implica no solamente la convivencia de culturas diferentes, la interacción, sino también el reconocimiento y el respeto de la diversidad» (Álvarez González, 2011: 2). Al optar por una pedagogía intercultural, se aprecian «las diferencias entre culturas como el resultado de una relación dinámica, no jerarquizante, entre dos entidades culturales que se dan sentido mutuamente» (Solís, 2012: 179). Alcanzar esta actitud crítica y reflexiva es lo que marca la diferencia entre la enseñanza de la cultura como información, que persigue la competencia cultural, y la enseñanza de la competencia intercultural.

Ahora bien, la cultura como «conjunto complejo que incluye el conocimiento, las creencias, arte o técnicas, moral, ley, costumbre y cualquier otra facultad y hábito que el hombre adquiere como miembro de la sociedad» (Tylor, 1977: 19) es un concepto casi inabarcable, por eso, en el ámbito educativo, requiere una mayor especificación que se ha resuelto con la identificación de los componentes que constituyen el contenido cultural que acompaña a las lenguas. Por tanto, para facilitar un acercamiento a este concepto, Miquel López y Sans Baulenas (1992) proponen una clasificación en tres grandes categorías:

- La Cultura con mayúscula, que abarca la noción tradicional de cultura, es decir, todo el compendio del saber artístico y literario, el saber de carácter geográfico, histórico y político, etc.

- La cultura con minúscula, que conforma el componente sociocultural y engloba todo aquello que los miembros de una sociedad «adscritos a una lengua y cultura, comparten y dan por sobreentendido» (Miquel López y Sans Baulenas, 1992: 17). 
- La kultura (con k), que comprende el saber, las costumbres o los usos que difieren del estándar cultural y no son compartidos por todos los hablantes, como el argot.

En esta clasificación, el humor encajaría dentro del componente sociocultural, en la categoría de cultura con minúscula y, "por sus características intrínsecas, implica no solo el dominio formal de la lengua extranjera, sino también el conocimiento de patrones y comportamientos culturales determinados» (Re, 2012: 117). El componente sociocultural, y por ende el humor, es el que da mayores problemas en la comunicación entre personas de diferentes procedencias, pues «donde el individuo actúa e interactúa con otros es en aquellos aspectos diarios de la cultura diaria, y es en estos donde surgen los malentendidos culturales»(García García, 2004). Las diferencias en las normas culturales y conversacionales, en los valores y en las expectativas de los interlocutores no solo suponen la interrupción del proceso comunicativo, sino que, además, pueden conllevar la aparición de malentendidos que den pie a la creación de prejuicios o estereotipos (Beyrich y Borowski, 2000; Oliveras Vilaseca y Llobera Cànaves, 2000; Merino Jular, 2010: 74-77), de ahí la importancia de incluir el humor en la enseñanza de segundas lenguas.

\subsection{El humor como elemento (inter)cultural en la enseñanza de ELE}

Este estudio se centra en la disparidad existente entre la cultura espanola y la japonesa y pretende analizar, entre otras cuestiones, la atención que los manuales analizados prestan a las formas de verbalizar el humor en español. Evidentemente, la cultura española o el humor español no son entes diferenciados, estáticos y comunes a todo hispanohablante, pues ni la cultura es homogénea entre países que comparten la misma lengua, ni lo es dentro de la misma nación, y lo mismo podría aplicarse a la cultura y el humor japoneses. Pero es una realidad que en un encuentro entre hablantes de español y de japonés, el uso del humor o la ironía podría llegar a desencadenar malinterpretaciones en el mensaje o en las intenciones de los hispanohablantes, principalmente, porque los japoneses evitan el humor que convierte en víctima al objeto del chiste; por eso, este tipo de humor no es habitual en sus interacciones orales o, al menos, no de forma tan directa como lo es en occidente (Wells, 1997; Liao y Abe, 2001: 192).

Al ser un aspecto sociocultural tan distintivo entre las diferentes lenguas y culturas y al tener tanto peso en la interacción oral, tanto el Plan Curricular del Instituto Cervantes (PCIC) como el Marco Común Europeo de Referencia para las Lenguas (MCER) recomiendan incluir el humor entre los saberes culturales, habilidades y actitudes que se deben trabajar en la enseñanza de lenguas. Pero, ¿siguen los manuales de ElE empleados en Japón 
estas recomendaciones? El análisis que se presenta a continuación pretende contestar a esta pregunta.

\section{Metodología}

La metodología empleada en nuestro análisis de manuales se podría definir como exploratoria-cuantitativa-interpretativa, dado que sigue un procedimiento muy claro: una vez revisado el material en busca del contenido cultural y humorístico (exploratoria), se rellena una plantilla de análisis anotando las referencias culturales en la categoría correspondiente (cuantitativa) $\mathrm{y}$, finalmente, se procede a discutir los resultados y deducir las conclusiones pertinentes a partir de los datos obtenidos (interpretativa). Esta metodología mixta, inicialmente descrita por Grotjahn (1987), es de las más usadas en la lingüística aplicada y, especialmente, en el análisis de materiales (Areizaga Orube, 2002; Santamaría Martínez, 2008; Álvarez Baz, 2012).

La plantilla de análisis (véase anexo) se confeccionó a partir de modelos de análisis utilizados en otros estudios de manuales (Fernández López, 2004; Álvarez Baz, 2012), aunque fue especialmente útil el modelo de plantilla propuesto por Santamaría Martínez (2008: 93). No obstante, hubo que simplificarla para adaptarla a las necesidades de un análisis que se centra en tres elementos. Así, se contabilizan las actividades que profundizan en los componentes culturales, las que desarrollan la competencia intercultural y las que contienen y trabajan el registro humorístico.

En primer lugar, se analizan los elementos socioculturales, que se han clasificado atendiendo a la distinción del apartado 5.1.1.2. El conocimiento sociocultural del MCER (2002: 100-101), a investigaciones previas sobre manuales ya mencionadas y a la distinción de C/c/kultura (Miquel López y Sans Baulenas, 1992: 4), de tal manera que en la plantilla se registran 8 categorías con sus respectivas subcategorías, sumando un total de 29 variables. Además, también se indica el ámbito geográfico al que hace referencia el contenido de cada actividad, tanto si se refiere solo a España o solo a Hispanoamérica, desde una perspectiva monocultural, si se refiere a la realidad hispanoparlante como conjunto, o si sigue un criterio más abierto englobando contenido del mundo de habla hispana y no hispana por igual.

En segundo lugar, para medir hasta qué punto estos manuales desarrollan la competencia intercultural, se tuvo en cuenta si el tratamiento del contenido cultural cumplía con alguno de los componentes de la lista elaborada por Álvarez González (2011: 5) para señalar los parámetros que definen un enfoque intercultural. Esta lista originalmente está compuesta por 13 componentes, sin embargo, para facilitar su medición, se determinó agruparlos en tres categorías según su afinidad: 
- Promoción de actitudes positivas.

- Equidad al tratar ambas culturas.

- Desarrollo de una actitud crítica.

En último lugar, se incluyó un apartado para medir todo aquello que esté relacionado con el registro cómico y humorístico. Se contabilizaron todas las muestras de humor que aparecen en cada manual y, luego, se estudió si hay un aprovechamiento didáctico o si es solo información facilitada por su interés cultural, como se haría al mostrar una viñeta de Rompetechos acompañada de una descripción del personaje y de su autor, Francisco Ibáñez. La plantilla incluye una variable para contabilizar aquellos casos en los que se incluya una explicación del trasfondo y la configuración pragmalingüística de la expresión verbal del humor.

Aunque somos conscientes de la dificultad que entraña extraer y medir estadísticamente un concepto expresado en forma de actividades y textos en manuales de enseñanza y, por tanto, del carácter interpretativo de algunos parámetros de este análisis, consideramos necesario acometer este tipo de trabajos para visibilizar y cuantificar la presencia de un aspecto que, como se ha señalado, debe formar parte del currículum de enseñanza de segundas lenguas.

3. Selección del corpus de datos: Manuales de ele usados en Japón

El estudio de todos los manuales de ele utilizados actualmente en Japón supera, de un lado, las posibilidades de análisis de este trabajo y, de otro, no garantiza el aporte de datos significativos para este estudio; por ello, hemos realizado una selección de manuales atendiendo a varios criterios que exponemos a continuación.

En primer lugar, consideramos que los manuales utilizados por el centro de referencia mundial en la enseñanza de ELE necesariamente debían formar parte de esta selección. Por ello, incluimos en nuestra lista inicial los libros de texto que el Instituto Cervantes de Tokio (IC Tokio) usa para sus cursos de ELE $^{1}$ ordenados por nivel del A1 al C2:

- Borobio Carrera, V. y Palencia del Burgo, R. (2012): Ele actual A1. Madrid, Grupo Editorial SM.

- Borobio Carrera, V. y Palencia del Burgo, R. (2012): Ele actual A2. Madrid, Grupo Editorial SM.

1 Esta lista de manuales ha sido elaborada a partir de los datos extraídos de la página web del IC Tokio <http://tokio.cervantes.es/es/cursos_espanol/matriculacion_condiciones. htm> [consulta: 22 de enero de 2018]. 
- Borobio Carrera, V. y Palencia del Burgo, R. (2012): Ele actual B1. Madrid, Grupo Editorial SM.

- Corpas, J.; Garmendia, A.; Sánchez, N. y Soriano, C. (2013): Aula internacional 4. Nueva Edición B2.1. Barcelona, Editorial Difusión.

- Natal, E.; Díez Francisco, M.C.; Buitrago, A.; Martín, M.S.; Prieto, J.M.; Fernández, J.; Castillo, M.; Borrego, I. y Núñez, B. (2008): Español lengua viva 4. Madrid, Santillana Educación.

- Del Mazo de Unamuno, M.; Muñoz Pérez, J.; Ruiz Mena, J. y Suárez Prieto, E. (2012): Nuevo prisma C2. Madrid, Editorial Edinumen.

Estos manuales tienen en común dos características: la primera es que han sido creados y editados por autores y editoriales occidentales, y la segunda es que no están dirigidos a estudiantes japoneses en particular, sino a estudiantes de ELE en general.

De ahí, que el segundo criterio de búsqueda que ha guiado esta selección haya sido precisamente la especificidad de los manuales, es decir, que hubieran sido elaborados específicamente para alumnos japoneses, con independencia de la nacionalidad de sus autores. Ahora bien, para garantizar que estos manuales cumplieran con la condición de estar actualizados, se determinó elegir solo los que se hubieran editado a partir de 2006, después de la publicación del PCIC, documento de referencia para una descripción de la enseñanza de ELE. Además, al revisar manuales posteriores a esta fecha, se podría comprobar si estos manuales siguen sus directrices y las sugeridas por el MCER. Nuestra búsqueda, llevada a cabo en la red, así como en catálogos de bibliotecas europeas y americanas, dio como resultado la siguiente lista de manuales para estudiantes japoneses elaborados por profesores de universidades japonesas en cooperación con autores españoles, que no incluye los ya descatalogados:

- De Prada, M.; Marcé, P.; Rosa de Juan, C.; Salazar, D. y UGhida, M. (2009): Español ya: Curso de español para japoneses. Madrid, Edinumen (nivel A1).

- Puebla, C. y Nishikawa, T. (2010): Primeros pasos en español. Madrid, SGEL (nivel A1).

- Sánchez Pérez, A. y Sensui, H. (2011): En directo Japón 1. Madrid, SGEL (nivel A1).

- Sánchez Pérez, A. y Sensui, H. (2012): En directo Japón 2. Madrid, SGEL (nivel B1).

Por último, a todos los resultados obtenidos atendiendo al primer y al segundo criterio, se aplicó un tercero que emana de la idea que se ha venido sosteniendo en este trabajo: la complejidad en la identificación y la expresión del humor. Esta complejidad exige un dominio de la lengua y de la cultura 
suficiente como para captar matices, dobles sentidos e inferir significados, algo que solo se adquiere a partir de niveles intermedios, como señala el PCIC. Al descartar los manuales de nivel inicial, el corpus se limitó a cinco de los manuales recogidos en las listas anteriores:

- B1: En directo Japón 2 (específico para estudiantes japoneses).

- B1: Ele actual B1.

- B2.1: Aula internacional 4.

- C1: Español lengua viva 4.

- C2: Nuevo prisma C2.

Esta selección de manuales nos ha permitido realizar un estudio detallado comparando ampliamente la forma de trabajar los contenidos y aspectos socioculturales, la competencia intercultural y el humor, verbal y no verbal. Los resultados derivados del análisis se presentan a continuación organizados en torno a cuatro cuestiones que se corresponden con los objetivos que establecimos en la introducción a este trabajo:

a) ¿Cómo se integra el componente cultural en los manuales? Con esta pregunta pretendemos dilucidar si el componente cultural se introduce en actividades destinadas a desarrollar las cuatro destrezas o si, por el contrario, los manuales dedican un espacio exclusivo a este contenido.

b) ¿Qué tipo de contenidos culturales se abordan en estos manuales? Atendiendo a la clasificación de cultura establecida por Miquel López y Sans Baulenas (1992), a la que hicimos mención en el apartado 1.1, descubriremos los contenidos culturales a los que se presta más atención y comprobaremos el ámbito geográfico al que se circunscriben estos contenidos.

c) ¿Se fomenta tanto la competencia cultural como la intercultural? El análisis desvelará si además del desarrollo de la competencia cultural, que dota a los estudiantes de conocimiento sobre la cultura meta, los manuales van un paso más allá y promueven un espíritu crítico hacia la cultura meta y las culturas de otros países, incluyendo la suya propia.

d) Por último, nos centraremos en el humor. La pregunta en este caso sería doble, en primer lugar ¿hay un espacio para el humor como elemento cultural en los manuales del corpus? Y, en caso afirmativo, ¿se explican las estrategias más comunes para expresar humor en español?

\section{AnÁlisis de los manuales del CORPUS}

La inclusión o no de contenido cultural en los manuales del corpus y su interés por promover la competencia (inter)cultural responde, en gran medida, a los enfoques metodológicos adoptados; así, la metodología de los 
manuales empleados en el IC Tokio comparten un mismo interés comunicativo y su estructuración y contenido se corresponden con las directrices del MCER. En cambio, el manual enfocado a estudiantes de origen japonés difiere en cuestiones de formato y fondo (estructuración, metodología y contenido). Luis Blanco (2014) analiza manuales publicados en Japón para estudiantes universitarios japoneses de nivel principiante absoluto y resuelve que «se adscriben en su práctica totalidad a una enseñanza tradicional, en la que el estudio de la gramática predomina sobre otro tipo de contenidos». Estas diferencias, que comparte el manual analizado en este estudio, son reflejo de una mentalidad conservadora en la enseñanza de lenguas en ese país.

\section{1. ¿Cómo se integra el componente cultural en los manuales seleccionados?}

Ele actual B1 contiene doce unidades, cada una dividida en cinco secciones claramente diferenciadas. Los contenidos culturales se van integrando en las actividades de la segunda sección, orientadas a desarrollar las cuatro destrezas (comprensión y expresión oral y escrita), pero se concentran especialmente en la cuarta sección de cada unidad, denominada «Descubre España y América Latina», que contiene el grueso de la información cultural mayoritariamente del tipo cultura con minúscula. Además, la última unidad del manual, llamada precisamente «Interculturalidad», se dedica exclusivamente a contenidos culturales cuya naturaleza estudiaremos en el próximo apartado.

En Aula internacional 4, el componente cultural es el elemento conductor que comparten muchas actividades independientemente de los recursos que pretendan explotar. Pero, al igual que en el manual anterior, cada unidad incluye un bloque denominado «Viajar» que se dedica específicamente a este componente, exponiendo la realidad cotidiana y cultural de los países de habla hispana.

Por su parte, Español lengua viva 4 está dividido en doce unidades cuyo contenido abarca cinco aspectos: «Comunicación», «Gramática», «Vocabulario», «Cultura y Sociocultura» y «Textos». No quiere decir que estas secciones aparezcan de forma diferenciada en cada unidad, es decir, no encontraremos una sección encabezada por el epígrafe «Cultura y Sociocultura», sino que cada unidad contiene un conjunto de ejercicios que responden a cada uno de estos bloques, aunque no sigan un orden específico. No obstante, los autores han señalado mediante un icono el objetivo y la competencia que pretenden desarrollar en cada una de esas actividades. Así, las que persiguen promover el conocimiento cultural y sociocultural, y la conciencia intercultural vienen identificadas mediante un icono con las letras «Cs».

Aunque con nombres diferentes, las unidades de Nuevo prisma C2 también agrupan sus contenidos en distintos bloques: contenidos funcionales, 
contenidos lingüísticos, tipos de texto y léxico, componentes estratégicos y contenidos culturales. Con respecto a los contenidos culturales, llama la atención que cada unidad toca muchos más temas (entre cuatro y nueve) que los manuales anteriores (entre tres o cuatro por unidad). Dado que en Nuevo prisma C2 tampoco se marcan secciones atendiendo a los bloques de contenido, las actividades que trabajan la competencia intercultural y el contenido cultural se identifican con las etiquetas intercultura o cultura, respectivamente, aunque, incomprensiblemente, muchos referentes culturales no aparecen indicados con ninguna etiqueta. Así, por ejemplo, desde la primera unidad se dedican hasta ocho actividades a conocer el estilo románico rural de Palencia, o a describir la ciudad de Córdoba, sin embargo, esta información, que es evidentemente cultural, no aparece señalada como tal. Por este motivo, en este, y en el resto de manuales, se registraron todos los contenidos culturales sin tener en cuenta su etiquetado.

En directo Japón 2, a diferencia de los anteriores, es producto de la colaboración de autores españoles y japoneses y se creó específicamente para el alumnado nipón. Este manual ofrece una distribución temática y una metodología distinta a la propuesta por los cuatro manuales ya analizados. En solo 103 páginas, este manual incluye un total de veinticuatro unidades que repiten exactamente el mismo patrón estructuralista: se inician con un diálogo corto que introduce la nueva gramática, luego, sigue una explicación de dicha gramática con ejemplos aclaratorios y, finalmente, se sucede una batería de ejercicios que siempre son de rellenar huecos, de repetir modelos, de conjugar verbos y de contestar preguntas. El papel del profesor como mediador cultural y el del alumno como hablante intercultural es nulo. La función del docente, en caso de seguir fielmente el manual, se reduce a ejercer como corrector y guía, alguien que puede reformular las explicaciones del manual y dar más ejemplos o ayudar, pero poco más. Los estudiantes, por su parte, repiten las mismas estructuras hasta fijarlas en su memoria, explotando la competencia lingüística y gramatical, pero sin desarrollar suficientemente la competencia comunicativa e intercultural. Esto explica que solo se haya contabilizado una actividad de contenido cultural en todo el manual.

\section{2. ¿Qué tipo de contenidos culturales se abordan en estos manuales?}

\subsubsection{Análisis individualizado}

Ya comentamos en el apartado anterior que las unidades de Ele actual B1 distribuían los contenidos culturales en las actividades de la segunda sección, destinadas a practicar las distintas destrezas, y en la cuarta sección, «Descubre España y América Latina». En estas actividades se trabaja con el cine, con datos geopolíticos de España y Latinoamérica, con literatura 
hispana y foránea, se trata el tema de la alimentación, etc. Por tanto, la mayoría de los contenidos culturales pertenecen a la categoría Cultura con mayúscula. Pero la cultura con minúscula también tiene cabida, principalmente en la unidad 12, «Interculturalidad», que incluye temas como el lenguaje corporal, las convenciones sociales, la imagen estereotipada de los españoles en las guías turísticas, las costumbres, los valores y actitudes españolas o latinoamericanas, etc. En general, como se puede apreciar en la figura 1, el contenido cultural tiende a prestar más atención a la Cultura con mayúscula que al componente sociocultural.

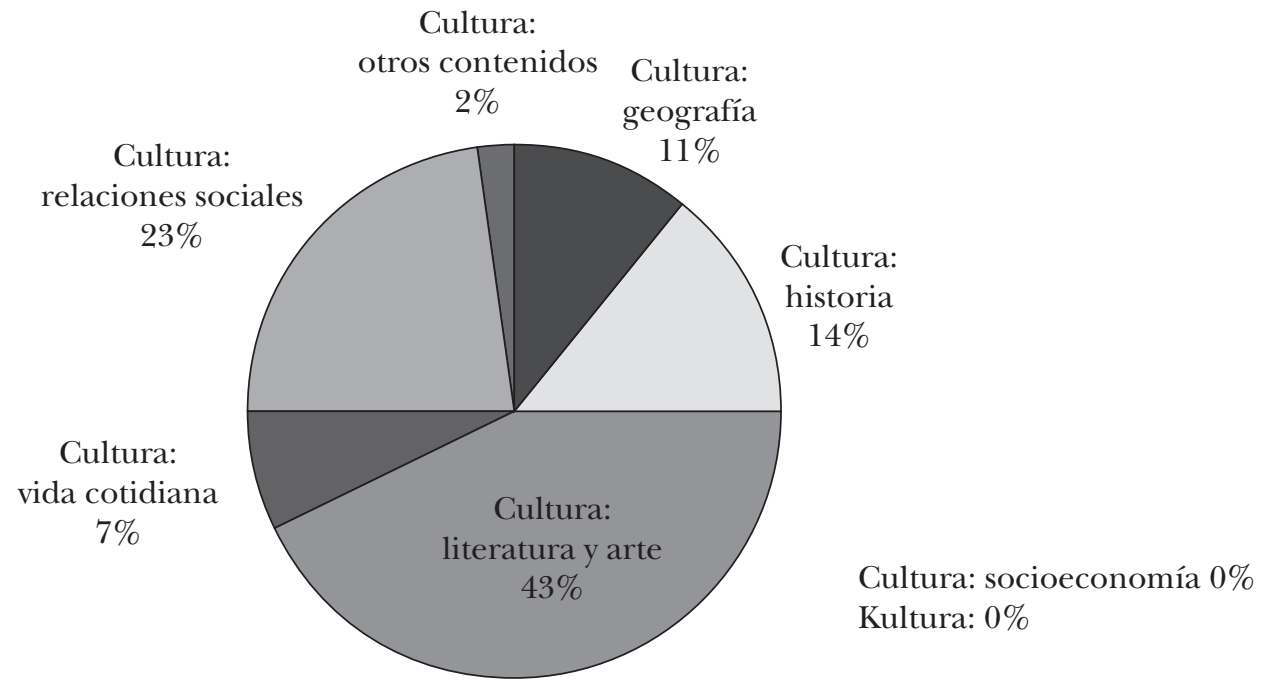

Figura 1. Contenido cultural de Ele actual B1.

Aula internacional 4, por otro lado, abarca, de forma algo más equilibrada, tanto la cultura con minúscula (ocio, medios de comunicación, turismo, valores y creencias, etc.), como la Cultura con mayúscula (obras literarias, arte o fiestas populares, entre otros). Al igual que en el manual anterior, y como se puede apreciar en la figura 2, no se ha podido identificar ningún elemento que haga referencia al registro informal, al argot o al uso coloquial de la lengua (kultura con $k$ ).

En Español lengua viva 4, la presencia del componente cultural es una constante hasta tal punto que, por primera vez, encontramos temas que podrían ser clasificados en todas las categorías establecidas por Miquel López y Sans Baulenas (1992), tal y como se recoge en la figura 3. Dentro de la cultura con minúscula, por ejemplo, se mencionan las malas condiciones de vida encontradas en determinadas zonas (específicamente la pobreza y la 


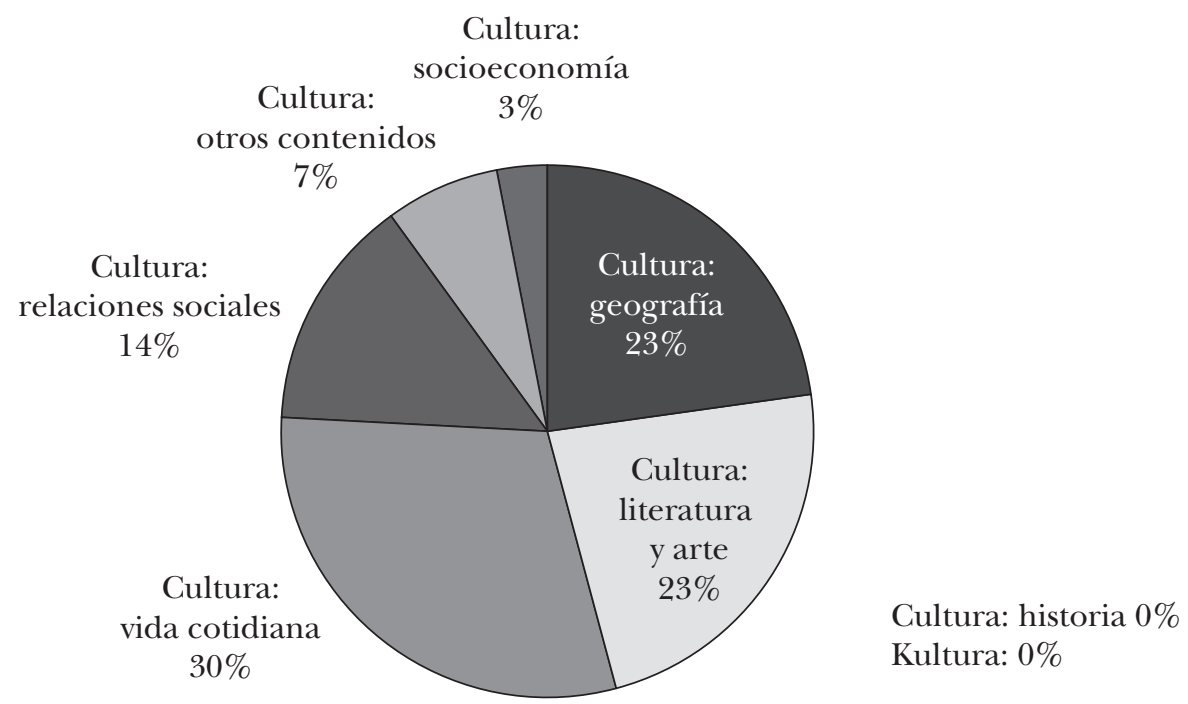

Figura 2. Contenido cultural de Aula internacional 4.

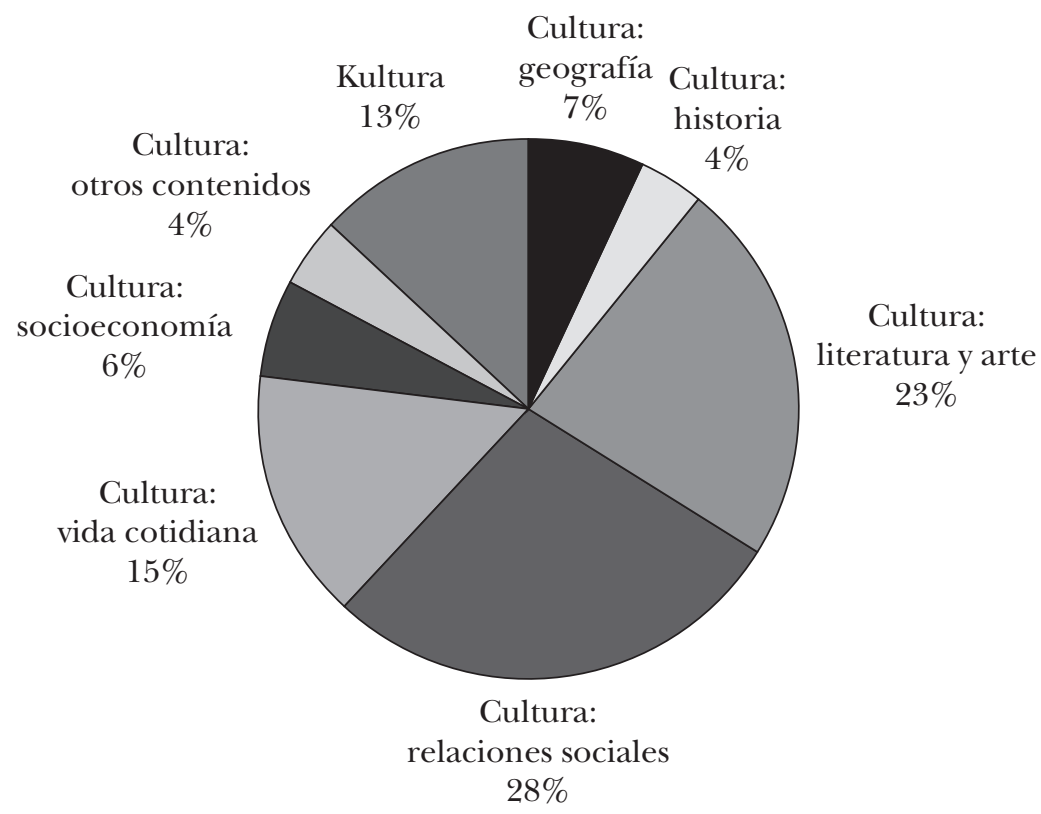

Figura 3. Contenido cultural de Español lengua viva 4. 
explotación infantil y los problemas para adquirir una vivienda), se habla de la influencia de los medios de comunicación y de la publicidad, de juegos y deportes populares, se describen políticas de empresa y sistemas políticos, etc. Cuando se tratan temas identificables como pertenecientes a la Cultura con mayúscula, se cumple con lo anticipado en el prólogo y se mencionan personajes, obras y lugares de interés tanto de España como de Hispanoamérica. En general la cobertura informativa es equilibrada entre los países hispanohablantes, aunque, es cierto que en demasiadas ocasiones la información se centra únicamente en España. En cuanto al contenido kultural, ausente en los anteriores, este manual incluye, por ejemplo, los saludos informales, los motes, las frases hechas y los modismos o algunas expresiones particulares que relacionan animales con adjetivos para describir a alguna persona: ser un gallina, ser un rata, ser un lince, estar como una cabra, etc.

Algunas facetas de la Cultura con mayúscula no tienen mucha presencia en Nuevo prisma C2. Así, por ejemplo, este manual no hace mucho hincapié en el cine, la música o el deporte — no contiene más de dos canciones, una de Rosana y otra de Mecano, y solo en una ocasión se habla de deporteaunque sí le da mayor cobertura al arte y la literatura con actividades relacionadas con la pintura o con distintos autores como Juan Ramón Jiménez, Julio Llamazares o Julio Cortázar, entre otros. Por el contrario, sí se muestra

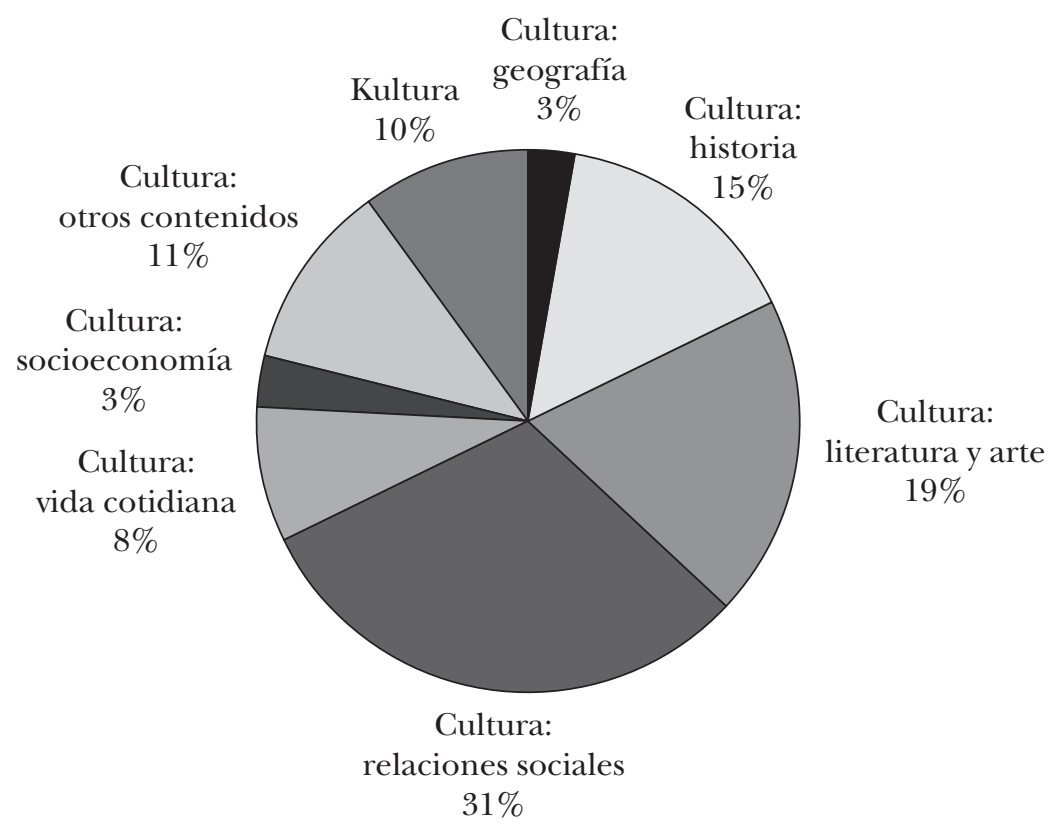

Figura 4. Contenido cultural de Nuevo prisma C2. 
más interés por cuestiones como los valores, las creencias, las actitudes y las convenciones sociales de la sociedad hispana, pertenecientes a la categoría cultura con minúscula, como se recoge en la figura 4. Posiblemente, esto se deba a que estos temas propician el debate y la discusión entre estudiantes de nivel avanzado. Dentro de esta categoría se incluyen actividades sobre la diversidad del español según circunstancias geográficas, sociales o generacionales, la estructura familiar y las relaciones de pareja en España, el lenguaje sexista y la controversia que lo envuelve, etc. En cuanto al ámbito geográfico que recibe más atención, este libro de texto lleva a cabo un evidente esfuerzo por recopilar el contenido cultural necesario para un buen conocimiento de España, consecuentemente, se centra mucho en este país y apenas se mencionan otros países de habla hispana.

Por último, y como cabría esperar dado su enfoque estructuralista, el manual elaborado para estudiantes japoneses, En directo Japón 2, contiene únicamente una actividad cultural que, dado el contenido geográfico que trata brevemente, podríamos clasificar dentro de la categoría de Cultura con mayúscula.

\subsubsection{Análisis comparativo}

Al examinar el ámbito geográfico al que se refieren las actividades culturales representadas, se pudo comprobar que todos los manuales, a excepción de En directo Japón 2, intentan hacer referencia a la realidad española e hispanoamericana, aunque todavía hay un claro favoritismo monocultural hacia España en la mayoría de los casos, destacando especialmente Nuevo prisma C2. El único manual que trata con equidad ambos ámbitos es Aula internacional 4. De hecho, este manual es el que más relaciones establece con otros países no hispanoparlantes (ya sea directamente o pidiendo a los alumnos que realicen esa conexión por ellos mismos), tal y como se muestra en la figura 5.

Por otro lado, el análisis individualizado evidencia que cada manual ha seguido un criterio propio en su selección de contenidos culturales, pues tocan temas muy diversos como vida cotidiana, historia, o relaciones sociales entre otros. Sin embargo, si nos detenemos en las grandes categorías, salvo en el caso particular del manual japonés, se observa que, a partir del nivel B1, los manuales incluyen más muestras de contenido cultural con minúscula (vida cotidiana y otras cuestiones sociales) que contenido Cultural con mayúscula (geografía, historia, literatura y arte) y, en segundo lugar, que el número de actividades relacionadas con ambas categorías aumenta progresivamente según avanzamos de nivel. Respecto a la presencia de kultura (registro coloquial, frases o modismos y argot juvenil), se aprecia que es muy escasa y se restringe a los manuales Español lengua viva 4 (C1) y Nuevo prisma C2, de 


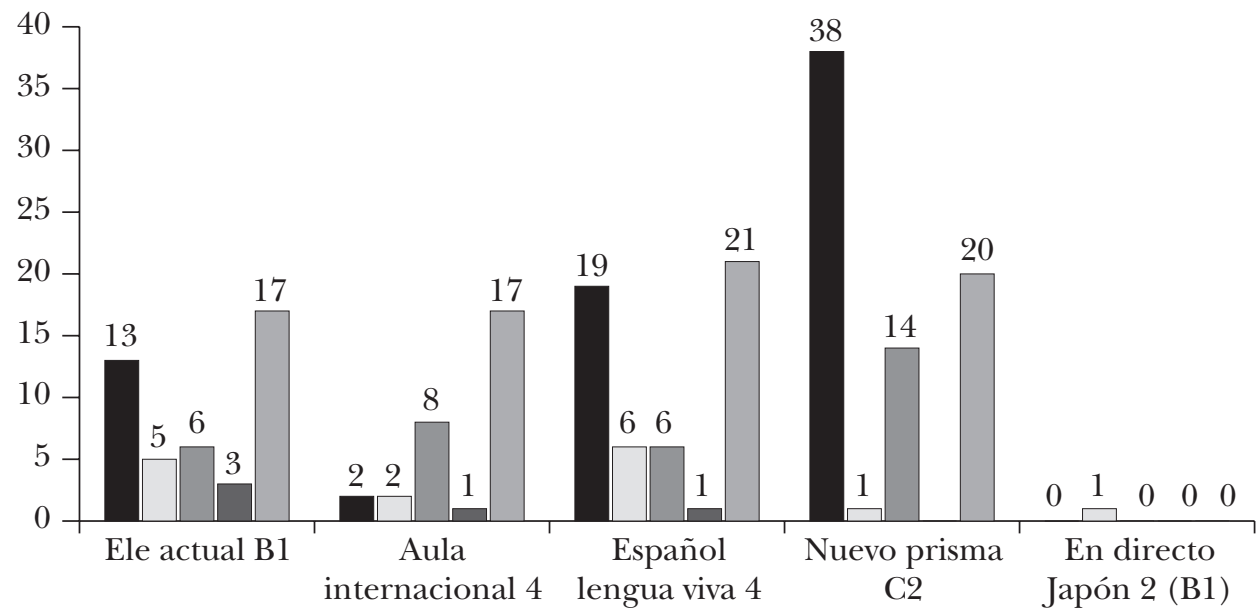

(B2.1)

(C1)

Solo España $\square$ Solo Hispanoamérica $\square$ Mundo hispano

$\square$ Otros $\square$ Mundo hispano y otros

Figura 5. Ámbito geográfico de las actividades culturales.

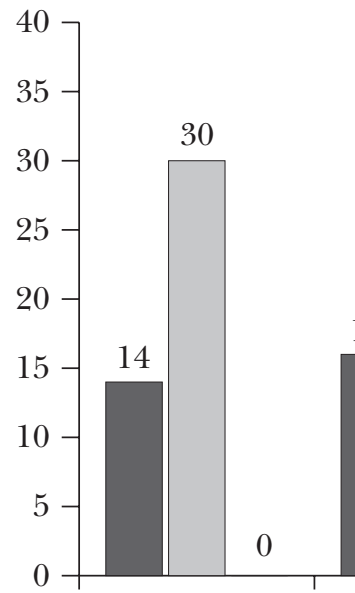

Ele actual B1

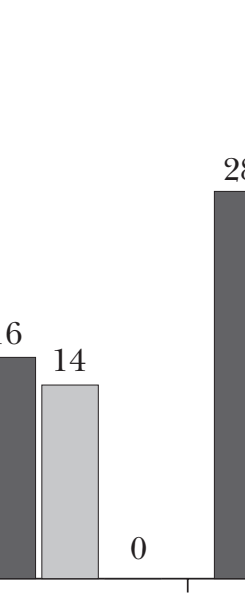

Aula

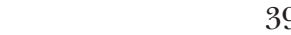

39

internacional 4 lengua viva 4

(B2.1)

(C1)

Cultura

Cultura

Kultura

Figura 6. Comparativa del contenido cultural de los manuales. 
niveles más avanzados. Este último, además, fue el único manual que presentó algunas muestras de lenguaje especializado — jurídico, en este caso-. Hasta cierto punto, podría justificarse la ausencia de este registro en los manuales iniciales, más centrados en el lenguaje común o estándar, pero no en los manuales de niveles intermedio e intermedio-avanzado.

\section{2. ¿̇Se fomenta tanto la competencia cultural como la intercultural?}

Al comprobar cuántas actividades incluyen contenidos culturales y cuántas desarrollan la competencia intercultural en cada manual, encontramos que la mayoría de los manuales incluyen pocas actividades con contenido cultural. Español lengua viva 4 es el único cuyas actividades culturales alcanzan la mitad de las totales, 53 de 105 (50,48\%). Además, vemos que la inclusión de este contenido cultural en los manuales no implica que se anime a los aprendientes a adoptar una actitud crítica con respecto a los temas que se incluyen, como se ilustra en la tabla 1 :

Tabla 1. Comparación de los contenidos (inter)culturales de los manuales del corpus seleccionado

\begin{tabular}{|l|c|c|c|c|c|}
\hline \multicolumn{1}{|c|}{ Actividades } & $\begin{array}{c}\text { Ele actual } \\
\text { B1 }\end{array}$ & $\begin{array}{c}\text { Aula int. 4 } \\
(\mathrm{B} 2.1)\end{array}$ & $\begin{array}{c}\text { Español } \\
\text { lengua viva } 4 \\
(\mathrm{C} 1)\end{array}$ & $\begin{array}{c}\text { Nuevo prisma } \\
\text { C2 }\end{array}$ & $\begin{array}{c}\text { En directo } \\
\text { Japón 2 (B1) }\end{array}$ \\
\hline Totales & 225 & 81 & 105 & 220 & 64 \\
\hline Culturales & 44 & 30 & 53 & 73 & 1 \\
$(19,5 \%)$ & $(37 \%)$ & $(50,48 \%)$ & $(33 \%)$ & $(1,5 \%)$ \\
\hline Interculturales & 19 & 8 & $\begin{array}{c}29 \\
(8,5 \%)\end{array}$ & $\begin{array}{c}26 \\
(9,9 \%)\end{array}$ & 0 \\
\hline
\end{tabular}

Esta tabla muestra que en Ele actual B1, el contenido cultural que se integra en la mayoría de las actividades dirigidas a practicar las cuatro destrezas se limita a enseñar la cultura como un elemento complementario al contenido lingüístico y solo en algunos casos se profundiza un poco más e incluso se desarrolla, en mayor o menor medida, la competencia intercultural. Sin embargo, en la sección «Descubre España y América Latina» de cada unidad, la información cultural que se facilita a los alumnos viene siempre acompañada de algunas preguntas para que los alumnos reflexionen sobre costumbres o situaciones similares en su propia sociedad.

Con respecto a Aula internacional 4, aunque se aprecia un mayor esfuerzo por incluir elementos culturales y ofrecer una cobertura informativa equilibrada de las culturas que se tratan, la competencia intercultural no se fomenta tanto como cabría esperar. Esta competencia requiere la reflexión 
y la discusión derivadas de la comparación de diferentes culturas y son pocos los casos en los que realmente se estimula esta actitud, aún cuando abundan las actividades que habrían servido perfectamente a este propósito.

En contraposición, y tal y como sucede con las actividades culturales, la tabla 1 muestra que Español lengua viva 4 es el manual que incluye más ejercicios destinados a fomentar la competencia intercultural con respecto al resto de actividades $(27,6 \%)$. En general, este manual trata una gran variedad de temas y en la mayoría de los casos se pide a los estudiantes que comparen la situación descrita con la que se vive en su propio país y que compartan sus conclusiones. Estas preguntas invitan a reflexionar sobre las lecturas y permiten desarrollar una actitud crítica. Además, en varias ocasiones se presentan brevemente algunas experiencias supuestamente reales con malentendidos culturales de otros estudiantes de español. Que se haga mención a estos malentendidos es una forma de preparar al alumnado como hablantes interculturales, pues se les anticipa que van a cometer errores y que se van a enfrentar a situaciones incómodas propiciadas por ellos mismos o por sus interlocutores extranjeros. No obstante, y a pesar de lo mucho que incorpora este manual en materia cultural y de haber demostrado tener muy en cuenta el desarrollo de la competencia intercultural, aún se pueden señalar algunas oportunidades que se podrían aprovechar para explotar aún más esta competencia. En ocasiones, se tratan algunos temas - como el sistema político, las familias, los problemas con la educación de los hijos o la obesidad infantil, etc.- desde una perspectiva meramente descriptiva y no se llega a profundizar ni se busca comparar con situaciones similares en otros países.

Por otro lado, a lo largo de Nuevo prisma C2 se intercalan muchas actividades que fomentan una cierta actitud crítica y positiva; es más, se hace reflexionar a los alumnos, directamente, sobre la relevancia del contenido sociocultural en el aprendizaje lingüístico. Entre otras, se incluyen actividades en las que los estudiantes comparan costumbres, creencias y valores propios del mundo hispano con las de su país de origen; además, también se contrastan aspectos socioculturales como las relaciones personales y sentimentales o los gestos; de hecho, el ejemplo que se incluye señala que en Japón mostrar el dedo meñique significa tener una novia secreta, mientras que en España significa estar muy delgado, prueba de la no universalidad del lenguaje corporal. Evidentemente, en este manual también encontramos, al igual que en los manuales anteriores, algunas oportunidades desaprovechadas para fomentar la competencia intercultural.

En directo Japón 2 se sitúa en el extremo opuesto al resto de manuales, con una atención nula a la competencia intercultural, algo esperable teniendo en cuenta su orientación metodológica. 


\section{3. ¿Hay un espacio para el humor en los manuales del corpus?}

La última parte de este estudio responde a nuestro interés por indagar en el tratamiento didáctico del humor como un elemento cultural característico y propio de toda sociedad. Nos parecía relevante explorar el contenido de los manuales para descubrir si este elemento, tan presente en las conversaciones diarias en el mundo hispano, también ocupaba un espacio en los manuales seleccionados. Sin embargo, no tratamos solo de identificar la presencia de enunciados humorísticos o de casos de humor gráfico, sino de comprobar si estos venían acompañados de explicaciones, textos o actividades destinados a interpretar y practicar recursos lingüísticos portadores de humor como la ironía, la metáfora, las hipérboles, etc. Nuestro estudio reveló que ninguno de los manuales analizados incluye muestras de humor, ni verbal ni gráfico, a excepción de Ele actual B1. Sin embargo, las tiras de Mafalda, las viñetas y las historietas cómicas en Ele actual B1 no persiguen explicar la expresión del humor en español, sino que sirven al mismo propósito que cualquier otro texto escrito: desarrollar la competencia lingüística mediante ejercicios con huecos a rellenar o con preguntas de comprensión, como se observa en la figura 7.

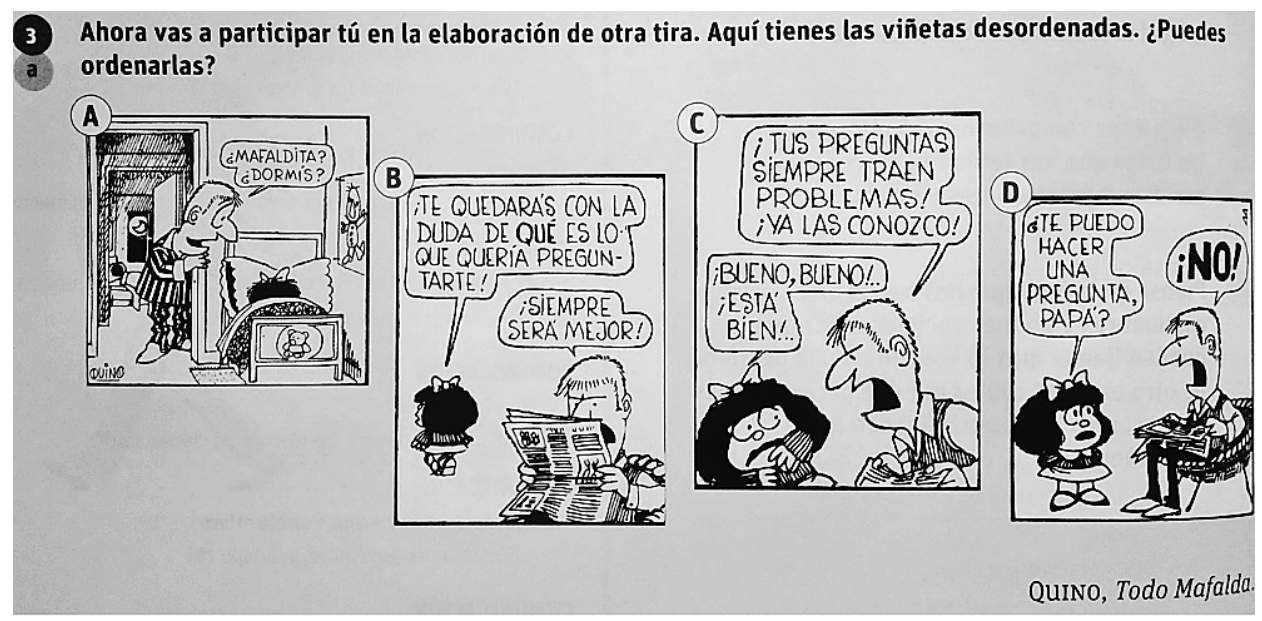

Figura 7. Tira de Mafalda en Ele actual B1 (2012: 158).

Evidentemente estos textos aportan más frescura y divertimento a las clases, pero se desaprovecha tanto aquí como en los demás manuales la oportunidad de enseñar algunas características de la expresión verbal del humor en español, algo lamentable teniendo en cuenta la relevancia del humor en el español coloquial y el enorme abanico de materiales disponibles. 


\section{Conclusiones}

El análisis llevado a cabo ha revelado que la presencia del contenido cultural difiere de un manual a otro, aunque se aprecian rasgos comunes. En primer lugar, de los manuales de ELE utilizados en el IC Tokio, dos incluyen en cada unidad una sección dedicada a contenidos socioculturales, mientras los otros dos señalan con iconos o etiquetas estos contenidos; además, las referencias socioculturales se intercalan a lo largo de distintas actividades. En cuanto al manual dirigido a estudiantes japoneses, En directo Japón 2, tal y como era esperable, no incluye información cultural alguna.

El tipo de contenido cultural también varía según el manual analizado y el nivel de referencia. En líneas generales, el espacio dedicado a las categorías cultura con minúscula y Cultura con mayúscula se incrementa a medida que aumenta el nivel de dominio de lengua al que se dirigen estos manuales. Por su parte, los contenidos kulturales escasean en los niveles intermedios: Ele actual B1 y Aula internacional 4; esta falta de homogeneidad en el tipo de contenidos culturales podría ser corregida con la inclusión de contenidos más variados atendiendo a la clasificación de Miquel López y Sans Baulenas (1992). Así, por ejemplo, en Aula internacional 4 y Ele actual B1 se podrían incluir muestras de registro informal o coloquial, dejando paso así a contenidos kulturales, y en Ele Actual B1 y Nuevo prisma C2 se podría disminuir la atención prestada únicamente a España en favor de un aumento de la presencia de referentes hispanoamericanos.

En cuanto a la competencia intercultural, aunque Español lengua viva 4 evidencia un número relativamente elevado de actividades que desarrollan esta competencia, todos los manuales se beneficiarían de la incorporación de actividades que despierten en los estudiantes la curiosidad por las diferencias culturales y promuevan el debate y la reflexión con una actitud abierta, crítica y flexible con el fin de borrar estereotipos y prejuicios.

Finalmente, de los resultados del análisis se desprende que el humor, un elemento sociocultural extremadamente particular en cada sociedad y de difícil interpretación y verbalización, no despierta el más mínimo interés en estos manuales de nivel intermedio e intermedio-elevado. Únicamente aparecen algunas muestras de humor en el manual de nivel más bajo de los estudiados, aunque sin ninguna información sobre la expresión lingüística, la formulación o el uso del humor en español.

En consecuencia, consideramos que, a pesar del enorme esfuerzo realizado en las últimas décadas por atender el componente cultural en los libros de textos de ELE, todavía pueden acometerse mejoras que redunden en beneficio del desarrollo de la competencia (inter)cultural y de la comprensión 
y expresión de un elemento tan complejo como el humor. Esta necesidad se torna aún más acuciante cuando estos manuales están dirigidos a un público culturalmente tan alejado del mundo hispanohablante como son los estudiantes japoneses. En este sentido, y como se ha señalado en estudios previos (Luis Blanco, 2014), los manuales elaborados específicamente para este alumnado tienen por delante un largo camino que recorrer si desean proporcionar algo más que competencia lingüística.

\section{BIBLIOGRAFÍA}

Álvarez BAz, A. (2012): El tratamiento de la interculturalidad en el aula con estudiantes de español sinohablantes. Tesis Doctoral. Granada, Universidad de Granada.

Álvarez González, S. (2011): «La relevancia del enfoque intercultural en el aula de lengua extranjera». Revista Nebrija de Lingüistica Aplicada a la Enseñanza de las Lenguas, 9.5, págs. 40-56.

Areizaga Orube, E. (2002): «El componente cultural en la enseñanza de lenguas: elementos para el análisis y la evaluación del material didáctico». Cultura y Educación, 14.2, págs. 161-175.

Beinhauer, W. (1973): El humorismo en el español hablado. Madrid, Gredos.

Bell, N.D. y Pomerantz, A. (2016): Humor in the Classroom: A Guide for Language Teachers and Educational Researchers. New York, Routledge.

Beyrich, D. y Borowski, C. (2000): «Malentendidos interculturales en la comunicación cotidiana». En AA.VV.: Propuestas interculturales. Actas de las VI Jornadas Internacionales TANDEM. Madrid, Edinumen, págs. 41-48.

Byram, M. (1997): Teaching and Assessing Intercultural Communicative Competence. Clevedon: Multilingual Matters.

Consejo de EuRopa (2002): Marco común europeo de referencia para las lenguas: aprendizaje, enseñanza, evaluación (trad. Instituto Cervantes). Madrid: Secretaría General Técnica del mec-Anaya.

Fernández López, M.C. (2004): «Principios y criterios para el análisis de materiales didácticos». En Sánchez Lobato, J. y Santos Gargallo, I.: Vademécum para la formación de profesores. Madrid, SGEL, págs. 715-734.

García García, P. (2004): «La cultura, ¿̇universo compartido? La didáctica intercultural en la enseñanza de idiomas». RedELE: Revista Electrónica de Didáctica ELE, 0 (en línea: <http://www.mecd.gob.es/educacion/mc/redele/revistaredele.html> [consulta: 22 de enero de 2018]).

GonzÁlez Verdejo, N. (2003): «Humor se escribe con "u” de universal. La risa como medio de acercamiento cultural». En Pérez Gutiérrez, M. y Coloma Maestre, J.: El español, lenguaje del mestizaje y la interculturalidad. Actas XIII Congreso AsELE. Madrid, CVC, págs. 346-357.

Grotjahn, R. (1987): «On the methodological basis of introspective methods». En Faerch, C. y Kasper, G.: Introspection in Second Language Research. Clevedon, Multilingual Matters, págs. 54-81. 
Instituto Cervantes (1997): Plan curricular del Instituto Cervantes. Niveles de referencia para el español. Centro Virtual Cervantes (en línea: <https://cvc.cervantes. es/ensenanza/biblioteca_ele/plan_curricular> [consulta: 22 de enero de 2018]).

Liao, C. y Abe, G. (2001): «A comparison of Taiwanese and Japanese appreciation of English jokes». Feng Chia Journal of Humanities and Social Sciences, 3, págs. 181-206.

Luis Blanco, J. (2014): «Tratamiento de la cultura en 26 manuales de ELE editados en Japón». Marco ELE. Revista de Didáctica Español Lengua Extranjera, 19 (en línea: $<$ https://marcoele.com> [consulta: 22 de enero de 2018]).

Merino Jular, E. (2010): «Culturas y creencias malentendidas dentro y fuera de la clase de L2 para inmigrantes adultos». Bellaterra Jounal of Teaching E् Learning Language Ė Literature, 3, págs. 70-87.

Miquel López, L. y Sans Baulenas, N. (1992): «El componente cultural: un ingrediente más en las clases de lengua». Cable, 9, págs. 15-21.

Oliveras Vilaseca, A. y Llobera CÀnaves, M. (2000): Hacia la competencia intercultural en el aprendizaje de una lengua extranjera. Estudio del choque cultural y los malentendidos. Madrid, Edinumen.

Paricio Tato, M.S. (2005): «La dimensión cultural en los libros de texto de lenguas extranjeras: pautas para su análisis». Glosas didácticas, 15, págs. 133-144.

RE, A. (2012): «Los monólogos humorísticos en la clase de E/LE: análisis lingüústico y didáctico». Lingue e Linguaggi, 7, págs. 117-130.

Santamaría Martínez, R. (2008): La competencia sociocultural en el aula de L2/LE: Una propuesta didáctica. Tesis doctoral. Madrid, Universidad Carlos III.

Solís, L.C. (2012): «La enseñanza de la competencia intercultural en el aula de E/LE: Consideraciones didácticas para programas de inmersión lingüístico-cultural (PILC)». Revista Nebrija de Lingüística Aplicada a la Enseñanza de las Lenguas, 11.6, págs. 174-192.

Tylor, E.B. (1977): Cultura primitiva. Madrid, Ayuso.

VAN Ek, P. (1986): Objectives for Foreign Language Learning. Strasbourg, Council of Europe.

Venezky, R.L. (1992): «Textbooks in schools and society». En Jackson, P.W.: Handbook on Research on Curriculum. New York, Macmillan.

Wells, M. (1997): Japanese Humour. Basingstoke, Macmillan. 
Anexo: Plantilla de ANÁlisis

\begin{tabular}{|c|c|c|}
\hline \multicolumn{3}{|c|}{ Contenidos socioculturales } \\
\hline \multirow{15}{*}{$\begin{array}{l}\text { cultura con } \\
\text { minúscula }\end{array}$} & \multirow{3}{*}{ Socio-economía } & Transporte \\
\hline & & Ocupación \\
\hline & & Economía \\
\hline & \multirow{4}{*}{ Vida cotidiana } & Horarios \\
\hline & & Alimentación \\
\hline & & Ocio \\
\hline & & Turismo \\
\hline & \multirow{7}{*}{$\begin{array}{l}\text { Relaciones } \\
\text { sociales }\end{array}$} & Condiciones de vida \\
\hline & & Relaciones personales \\
\hline & & Valores, creencias y actitudes \\
\hline & & El lenguaje corporal \\
\hline & & Convenciones sociales \\
\hline & & Estructura familiar \\
\hline & & Prácticas rituales \\
\hline & Otros contenidos & \\
\hline \multirow{10}{*}{$\begin{array}{l}\text { Cultura con } \\
\text { mayúscula }\end{array}$} & \multirow{5}{*}{ Literatura y arte } & Obras literarias \\
\hline & & Fiestas tradicionales \\
\hline & & Cine y música \\
\hline & & Pintura y escultura \\
\hline & & Arquitectura \\
\hline & \multirow{2}{*}{ Historia } & Personajes \\
\hline & & Acontecimientos \\
\hline & \multirow{3}{*}{ Geografía } & Clima \\
\hline & & Lugares de interés \\
\hline & & Medioambiente \\
\hline \multirow{4}{*}{ kultura con $\mathrm{K}$} & & Argot juvenil \\
\hline & & Registro coloquial \\
\hline & & Frases o modismos \\
\hline & & Cultura adolescente \\
\hline \multirow{3}{*}{$\begin{array}{l}\text { Ámbito } \\
\text { geográfico }\end{array}$} & & España \\
\hline & & Hispanoamérica \\
\hline & & Otros \\
\hline
\end{tabular}




\begin{tabular}{|l|l|}
\hline \multicolumn{2}{|c|}{ Competencia intercultural } \\
\hline \multirow{2}{*}{} & Promoción de actitudes positivas \\
\cline { 2 - 2 } & Equidad en el tratamiento de ambas culturas \\
\cline { 2 - 2 } & Actitud crítica \\
\hline Registro humorístico \\
\hline \multirow{2}{*}{} & Muestras de humor \\
\cline { 2 - 2 } & Aprovechamiento didáctico \\
\cline { 2 - 2 } & Profundización en expresión verbal del humor \\
\hline
\end{tabular}

